NASA Contractor Report 185131

AIAA-89-2334

\title{
Antiproton Powered Propulsion With Magnetically Confined Plasma Engines
}

Michael R. LaPointe

Sverdrup Technology, Inc.

NASA Lewis Research Center Group

Cleveland, Ohio

August 1989

Prepared for

Lewis Research Center

Under Contract NAS3-25266

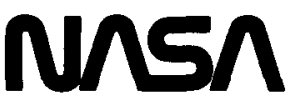

National Aeronautics and Space Administration 


\title{
ANTIPROTON POWERED PROPULSION WITH MAGNETICALLY CONFINED PLASMA ENGINES
}

\author{
Michael R. LaPointe* \\ Sverdrup Technology, Inc. \\ NASA Lewis Research Center Group \\ Cleveland, Ohio 44135
}

\begin{abstract}
Matter-antimatter annihilation releases more energy per unit mass than any other method of energy production, making it an attractive energy source for spacecraft propulsion. In the magnetically confined plasma engine, antiproton beams are injected axially into a pulsed magnetic mirror system, where they annihilate with an initially neutral hydrogen gas. The resulting charged annihilation products transfer energy to the hydrogen propellant, which is then exhausted through one end of the pulsed mirror system to provide thrust. The calculated energy transfer efficiencies for a low number density $\left(10^{14} \mathrm{~cm}^{-3}\right)$ hydrogen propellant are insufficient to warrant operating the engine in this mode. Efficiencies are improved using moderate propellant number densities $\left(10^{16} \mathrm{~cm}^{-3}\right)$, but the energy transferred to the plasma in a realistic magnetic mirror system is generally limited to less than $2 \%$ of the initial proton-antiproton annihilation energy. The energy transfer efficiencies are highest for high number density $\left(10^{18} \mathrm{~cm}^{-3}\right)$ propellants, but plasma temperatures are reduced by excessive radiation losses. Low to moderate thrust over a wide range of specific impulse can be generated with moderate propellant number densities, while higher thrust but lower specific impulse may be generated using high propellant number densities. Significant mass will be required to shield the superconducting magnet coils from the high energy gamma radiation emitted by neutral pion decay. The mass of such a radiation shield may dominate the total engine mass, and could severely diminish the performance of antiproton powered engines which utilize magnetic confinement. The problem is compounded in the antiproton powered plasma engine, where lower energy plasma bremsstrahlung radiation may cause shield surface ablation and degradation.
\end{abstract}

\section{Introduction}

Over the past few decades, spacecraft propulsion systems based on the combustion of chemical fuels have proven a reliable and satisfactory propulsion source. As ambitions in space continue to grow, however, the use of chenical fuels requires multiple rocket staging, large and expensive propellant-to-payload mass ratios, and increasingly complex orbital dynamics for intuitively simple missions. The limited energy available in chemical combustion is inadequate for several missions of interest, and the continued advancement of space exploration will require a commitment to develop new, advanced propulsion concepts.

- Member, AIAA 
Perhaps the most exotic reaction considered for advanced spacecraft propulsion is the annihilation of matter and antimatter. The energy released per kilogram of combined matter and antimatter is over 250 times the specific energy released in nuclear fusion, and over 8 orders of magnitude greater than the specific energy released in chemical combustion (1). Efficiently transferring this reaction energy to a propellant could provide high thrust and optimum exhaust velocities without the limitations imposed by chemical combustion energy or massive external power supplies. By tailoring the amount of annihilation energy required to perform a specific mission, spacecraft mass ratios' less than 5:1 may be obtained(2).

\section{Background}

All known elementary particles, with the exception of the photon and the $\pi^{0}$ and $\mu^{0}$ mesons, have antiparticle counterparts. An antiparticle has the same mass, spin, and lifetime as its complimentary particle, but opposite charge (if any) and opposite alignment between its spin and magnetic moment. The photon, $\pi^{0}$ meson, and $\mu^{0}$ meson are their own antiparticles. Stable elementary particles include the electron (positron), proton (antiproton), photons, and neutrinos.

When a particle and antiparticle meet they annihilate, liberating energy equal to twice the rest mass of either particle. This energy may be produced immediately as energetic gamma rays, or the annihilation process may produce intermediate particles which then decay or undergo further annihilation. The need to store the antimatter in a stable form for long duration space flight limits possible antimatter fuels to the positron and the antiproton.

\section{Electron-Positron Annihilation}

Direct annihilation of an electron with a positron proceeds via the reaction

$$
e^{-}+e^{+} \rightarrow 2 \gamma \quad\left(E_{\gamma}=0.511 \mathrm{MeV}\right)
$$

where each of the emitted $\gamma$-ray photons has an energy equal to half the sum of the rest mass plus kinetic energies of the electron-positron $\left(e^{-} e^{+}\right)$pair. An electron and positron may also undergo radiative capture to form a bound state of positronium (Ps) through the process

$$
e^{-}+e^{+} \rightarrow P s+h \nu
$$

The energetic $\boldsymbol{\gamma}$-rays are then released upon subsequent annihilation of the positronium.

The first serious consideration of antimatter annihilation energy for space travel centered on electron-positron annihilation. In 1953 Sanger ${ }^{(3)}$ proposed the concept of a "photon rocket", which was an attempt to utilize the energetic gamma rays either to provide direct thrust or to heat a propellant. However, engine efficiency using a directed $\gamma$-ray exhaust is limited by the inability to effectively collimate the energetic photons. Although the - -ray energy will not couple directly to a propellant, it could be absorbed by a refractory metal heat exchanger. A propellant is then heated as it passes through channels in the heat exchanger, with the hot gas exhausted through a standard nozzle to provide thrust. The storage density of positronium fuel is so low, however, that the required storage facility mass may overwhelm any potential benefit derived using electron-positron annihilation energy (4).

\footnotetext{
'The empty vehicle mass plus the propellant mass, divided by the empty vehicle mass.
} 


\section{Proton-Antiproton Annihilation}

Antiproton annihilation enjoys several advantages over positron annihilation as an energy source for spacecraft propulsion. The higher rest mass energies of the proton and antiproton yield a total of 1877 $\mathrm{MeV}^{\ddagger}$ per annihilation event, compared with $1.02 \mathrm{MeV}$ released by electron-positron annihilation. As discussed below, a significant fraction of the proton-antiproton $(p \bar{p})$ annihilation energy appears in the kinetic energy of charged particles, which may be collimated for direct thrust or used to heat a propellant more effectively than the gamma radiation released in electron-positron annihilation.

Antiprotons and positrons may be cooled and combined to form antihydrogen ${ }^{(5)}$. Although it remains a technical challenge, it may be possible to further condense the antihydrogen to form antihydrogen crystals $(4,5)$. Several schemes have been proposed to confine the antihydrogen "ice" using magnetic, electrostatic, or electromagnetic traps ${ }^{(5,6)}$, yielding a much higher storage density for the antiproton fuel. Electromagnetic radiation may be used to detach antihydrogen molecules or atoms from the surface of the crystal and ionize the antihydrogen into positrons and antiprotons ${ }^{(6)}$. The antiprotons may be guided with electric and magnetic fields to a reaction chamber, where they annihilate with protons to provide propulsive energy. The positrons, stripped from the antihydrogen atoms, could be used to provide additional power for auxiliary spacecraft components ${ }^{\left({ }^{(}\right)}$.

Protons and antiprotons may undergo direct annihilation, or at low energies may form bound states of protonium (Pn) leading to subsequent annihilation ${ }^{(7)}$. Protonium may be formed as a result of radiative capture

$$
p+\bar{p} \rightarrow P n+h \nu
$$

or by rearrangement collisions in a hydrogen gas

$$
\bar{p}+H \rightarrow P n+e^{-}
$$

Cross sections for each process are plotted against center-of mass energies in Figure 1. At low energies, $p \bar{p}$ annihilation proceeds via rearrangement collisions, while direct annihilation dominates at energies above roughly $10 \mathrm{eV}$. Radiative capture cross sections are not significant at energies above a fraction of an $\mathrm{eV}$, and will be neglected.

Figure 1: Conlomb corrected cross sections for proton-antiproton direct annihilation $\left(\sigma_{o p}\right)$, radialive eapture $\left(\sigma_{r}\right)$, and rearrangemeat collisions $\left(\sigma_{, H}\right)$, normalized to atomic eross section $\left(x r_{0}^{2}=8.7974 x\right.$ $10^{-17}$ ). Adapled from Morgan and Eughes $(n$.

$1 \mathrm{MeV}=10^{6} \mathrm{eV}=1.6 \times 10^{-13}$ Joules

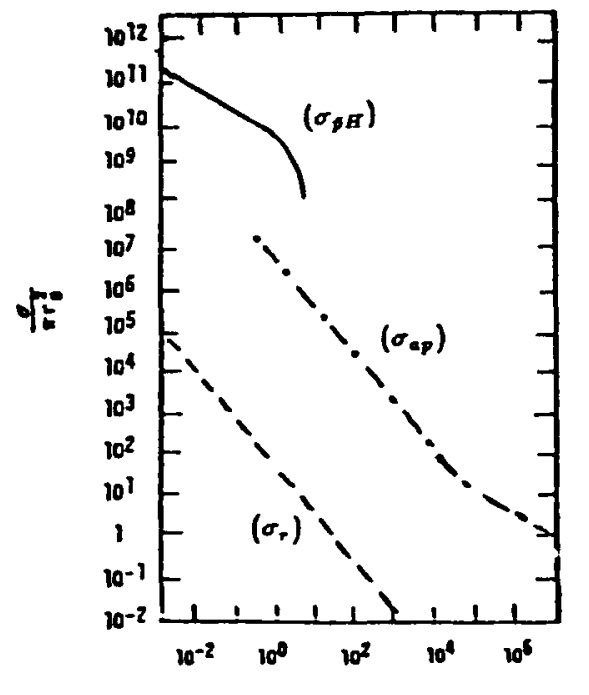

D-H or D-D Conter of hass Rinetic Energy (ev) 


\begin{tabular}{|c|c|}
\hline 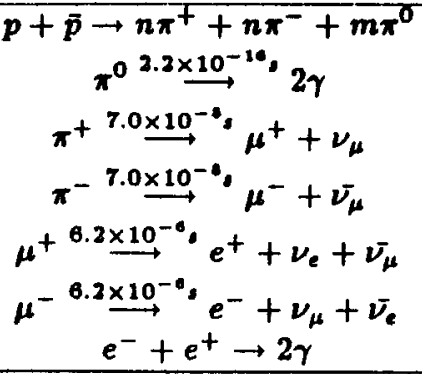 & $\begin{array}{c}E_{\gamma} \simeq 130-300 \mathrm{MeV} \\
\overline{K E}\left(\pi^{ \pm}\right) \simeq 250 \mathrm{MeV} \\
\overline{K E}\left(\mu^{ \pm}\right) \simeq 192.3 \mathrm{MeV} \\
\overline{K E}\left(e^{+}\right) \simeq 100 \mathrm{MeV} \\
\overline{K E}\left(e^{-}\right) \simeq 100 \mathrm{MeV} \\
E_{\gamma}=0.511 \mathrm{MeV}\end{array}$ \\
\hline
\end{tabular}

Table 1: Proton-Antiproton Annihilation Scheme.

The general proton-antiproton annihilation scheme is presented in Table 1. Each of the three charged pions has a rest mass energy of $139.6 \mathrm{MeV}$, and each of the two neutral pions has a rest mass energy of $135.0 \mathrm{MeV}$; thus a total of $689 \mathrm{MeV}$ is contained in pion rest mass energies. The average kinetic energy of the charged pions is $250 \mathrm{MeV} /$ pion $^{(8)}$, thus $750 \mathrm{MeV}$ or about $40 \%$ of the annihilation energy resides in charged pion kinetic energy. With a kinetic energy of $250 \mathrm{MeV}$, the pion is travelling at $93 \%$ the speed of light and relativistic effects extend its lifetime from 26 ns at rest to about 70 ns. Unless acted upon, it will travel almost 21 meters before it decays.

The remainder of the annihilation energy resides in the kinetic energy of the neutral pions, roughly $220 \mathrm{MeV} /$ pion. At this energy the neutral pion lifetime is extended from $8.4 \times 10^{-17}$ seconds to about $2.2 \times 10^{-16}$ seconds, and it will travel $6.6 \times 10^{-8}$ meters before decaying. Upon decay each neutral pion produces two energetic gamma rays, each with energies between 130 and $300 \mathrm{MeV} / \gamma$.

The decay of the $\pi^{+}$or $\pi^{-}$meson liberates an amount of energy equal to their rest mass energy and kinetic energy combined, or roughly $390 \mathrm{MeV} /$ pion. The $\pi^{+}$decays into a charged muon $\left(\mu^{+}\right)$ and a mu neutrino $\left(\nu_{\mu}\right)$. The $\mu^{+}$has a rest mass energy of $105.7 \mathrm{MeV}$ and an average kinetic energy of $192.3 \mathrm{MeV} /$ muon $^{(8)}$, leaving the neutrino with roughly $90 \mathrm{MeV}$. The energetics are the same for the decay of a $\pi^{-}$into a $\mu^{-}$and an anti $\mu$-neutrino $\left(\bar{\nu}_{\mu}\right)$. The total kinetic energy of the three charged muons created by the decay of the three charged pions is thus $577 \mathrm{MeV}$, or about $31 \%$ of the initial $p \bar{p}$ annihilation energy.

A charged muon with a kinetic energy of $192.3 \mathrm{MeV}$ is travelling at $94 \%$ the speed of light, and its lifetime is increased from $2.2 \times 10^{-6}$ seconds to $6.2 \times 10^{-6}$ seconds. Unless acted upon, the muon will travel almost 1800 meters before it decays. The neutrinos, travelling at the speed of light, will carry their energy out of the system.

The $\mu^{+}$decays into a positron, an $e$ neutrino $\left(\nu_{e}\right)$, and an anti- $\mu$ neutrino $\left(\overline{\nu_{\mu}}\right)$, while the $\mu^{-}$ decays into an electron, a $\mu$ neutrino, and an anti-e neutrino $\left(\bar{\nu}_{e}\right)$. The total energy available from the charged muon decay is about $300 \mathrm{MeV} /$ muon. Of this energy, $0.511 \mathrm{MeV}$ goes into creating the electron or positron, about $100 \mathrm{MeV}$ into the electron or positron kinetic energy ${ }^{(8)}$, and the remainder to the non-interacting neutrinos. Thus the decay of the three charged muons arising in the $p \bar{p}$ annihilation scenario will provide roughly $300 \mathrm{MeV}$ in the form of kinetic energy of the three electrons/positrons, corresponding to $16 \%$ of the initial proton-antiproton annihilation energy. The electrons and positrons are stable against decay, but can annihilate with one another to produce $0.511 \mathrm{MeV} \gamma$-rays, the ultimate end of the $p \bar{p}$ annihilation process. 


\section{Antiproton Powered Plasma Engine}

Several antiproton powered rocket designs have been suggested, ranging from the low thrust, high specific impulse pion rocket to the high thrust, low specific impulse solid and gas core thermal engines $^{(5)}$. The former concept is restricted to interstellar or deep space missions which require relativistic exhaust velocities, while the latter concepts are limited in performance by thermal constraints on material walls. A design which minimizes material constraints while providing a range of operating parameters is the antiproton powered plasma rocket, which utilizes magnetic mirror fields to contain both the charged annihilation byproducts and the ionized propellant. Morgan ${ }^{(\theta)}$ has sketched a design for a pulsed heavy ion plasma engine, in which antiprotons annihilate with nucleons in a heavy atom nucleus to produce energetic charged nuclides and particles. The charged byproducts are confined by external magnetic field coils and collisionally transfer their energy to the remaining heavy atoms, forming a magnetically confined plasma. The magnetic mirror field is then relaxed at one end and the plasma escapes to provide thrust. By tailoring the antiproton and propellant injection rates, it is possible to achieve a variety of thrust and specific impulse values with a single engine design.

The efficiency of the pulsed heavy ion plasma engine is limited by the amount of energy residing in the kinetic energy of the charged annihilation byproducts, estimated to be less than $20 \%$ of the initial annihilation energy ${ }^{(9)}$; of this amount some fraction less than unity will be transferred to the heavy ion propellant. Engine life will be limited by radioactive byproduct decay, neutron radiation damage, and neutral fragment sputtering of surrounding material. If instead the charged byproducts produced by antiproton annihilation in hydrogen could be contained and their energy coupled to a hydrogen plasma, a variable thrust, variable $I_{\text {sp }}$ engine could be designed without the disadvantages of heavy atom annihilation. With this goal in mind, a computer model was constructed to predict the performance of an antiproton powered, magnetically confined hydrogen plasma engine.

\section{Pulsed $\bar{p}$-H Rocket Model}

The system to be modeled is dynamic and complex. On one time scale, antiprotons annihilate with an initially neutral hydrogen gas to produce relativistic, charged pions. The pions traverse the hydrogen propellant and transfer some amount of energy before decaying into charged, relativistic muons over a time scale of several nanoseconds. The energetic muons interact with the hydrogen over microsecond time scales before decaying into extremely relativistic electrons and positrons. The electrons and positrons in turn give up energy to the hydrogen over a time scale corresponding to the plasma confinement time, typically a few milliseconds. The electrons and positrons are stable, although positrons may be lost due to annihilation with plasma electrons. Strong magnetic fields are used for confinement and stability, but even so particles will be continually lost from the system. Thus at any given time the ensemble will consist of protons, antiprotons, plasma electrons, hydrogen atoms, pions $( \pm)$, muons $( \pm)$, decay electrons, and positrons, evolving and interacting on time scales which may differ over six orders of magnitude.

To simplify the system modeling, the $\pi^{+}$and $\pi^{-}$mesons are not distinguished, and are assumed to have an initial kinetic energy equal to the average distribution kinetic energy of $250 \mathrm{MeV} /$ pion. Similarly, no distinction is made between the charged muons, which are assumed to have an initial kinetic energy of $192 \mathrm{MeV} /$ muon. Muon decay produces electrons and positrons, and it is assumed that half of the electron/positron number density consists of electrons, the other half of positrons, each with an initial kinetic energy of $100 \mathrm{MeV} / \mathrm{e}^{ \pm}$. This separation allows an estimate to be made of positron depletion due to annihilation with plasma electrons. The assumption of indistinguishable particles is valid since Coulomb interactions and energy exchange rates are proportional to even powers of the particle charge. The possibility of relativistic $\pi^{+} \pi^{-}$or $\mu^{+} \mu^{-}$annihilation occuring in 
low pion or muon number densities over their respective lifetimes is remote and not included in the model. The use of initial average kinetic energies is somewhat crude, but a comparison of energy loss rates calculated over the range of experimentally observed particle kinetic energies indicates the error introduced by this approximation is negligible ${ }^{(10)}$.

Rate equations ${ }^{(10)}$ are used to evaluate the antiproton and charged annihilation byproduct number densities at each time step. Both $\bar{p} H$ rearrangement collision annihilation and $p \bar{p}$ direct annihilation cross sections are included in the antiproton annihilation rate calculations. Positron annihilation with ambient plasma electrons is included in the model. Relativistic forms of the energy loss equations derived by Bethe-Bloch ${ }^{(11)}$ and Sivukhin ${ }^{(12)}$ are used to estimate the energy transferred by the relativistic particles to the hydrogen propellant. Bremsstrahlung and synchrotron radiation may be neglected for the energetic pions and muons ${ }^{(10)}$, but are important energy loss mechanisms for the relativistic electrons and positrons and are included in the simulation. Plasma synchrotron radiation is neglected, but plasma bremsstrahlung radiation, a dominant energy loss mechanism at higher temperatures and plasma number densities, is evaluated at each time step and the plasma energy adjusted accordingly. Deexcitation and recombination energy losses, which may appear as the plasma is heated from an initially neutral to fully ionized state, are small and are not included in the model ${ }^{(10)}$.

The simulation treats all similar particles created during a given time step as an identical group; thus, all pions created during the $n^{\text {th }}$ time step $\Delta t$ have the same energy, lose the same amount of energy to the hydrogen propellant, and decay at the same time. Similar arguments hold for the muon and electron/positron distributions. Hence at any given time there will be $\left[\frac{70 n t}{\Delta t}\right]$ groups of pions in the system, $\left[\frac{\mathrm{B} \cdot 2 \mu s}{\Delta t}\right]$ groups of muons, and a continually growing number of electron/positron groups built up as the muon groups decay. Each group is followed through the system until they decay (for pion and muon groups) or until the run is completed (for the electron/positron groups). This bookkeeping procedure allows reasonable estimates to be made of the particle-propellant interactions without requiring excessive computer time. Details of the code structure are given in reference 10 .

\section{Particle Confinement}

Magnetic fields are used to constrain the charged annihilation byproducts and confine the hydrogen plasma away from any material walls. The magnetic mirror system (Figure 2) is assumed to consist of solenoidal current coils to produce a longitudinal magnetic field and mirror field coils at each end of the system to provide particle containment and overall MHD stability ${ }^{(13)}$. Antiprotons are injected axially along magnetic field lines and the neutral hydrogen propellant is injected radially across the field lines. Once the plasma is heated, one end of the magnetic mirror system is relaxed, forming a magnetic nozzle and allowing the plasma to escape.

The minimum magnetic field strengths required to contain the system of relativistic particles and plasma propellant are calculated by balancing the magnetic field pressure and plasma/particle kinetic pressure,

$$
\frac{B^{2}}{8 \pi}=\sum_{j}(n k T),
$$

where $\mathbf{n}$ is the number density and $(\mathrm{kT})$ is the temperature of the $j^{\text {th }}$ species, and the summation extends over all particle species present in the system. An additional constraint on minimum field strengths is imposed by requiring the relativistic particle gyroradii to be much smaller than the reaction chamber dimensions, yielding a minimum field strength of about 10 Tesla for typical reaction 


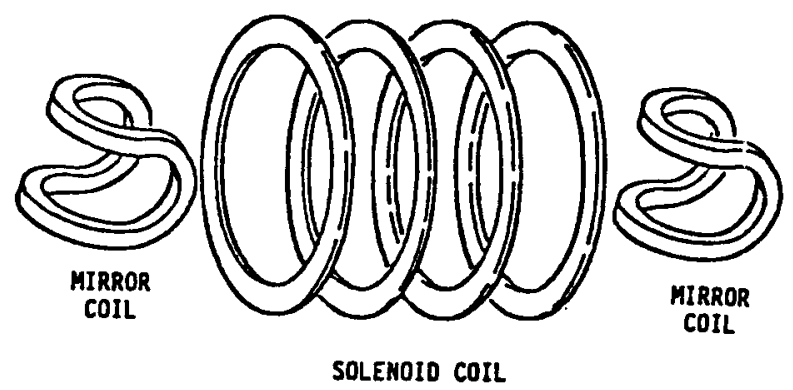

(a)

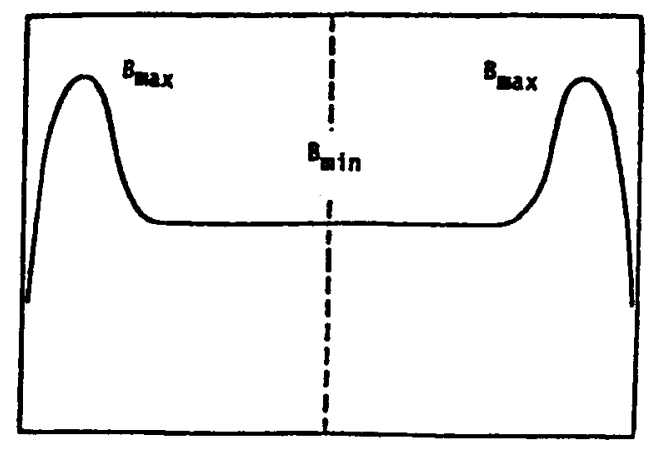

(b)

Figure 2: Magnetic Mirror System. (a) Field Coils. (b) Field Strength.

chamber dimensions ${ }^{(10)}$.

The probability that a charged particle will be lost from the magnetic mirror system is given by ${ }^{(14)}$

$$
P=1-\left(\frac{R_{m}-1}{R_{m}}\right)^{1 / 2}
$$

where $P$ is the probability of escape and $R_{m}$ is the magnetic mirror ratio, the ratio of the maximum to minimum magnetic field strengths. For a uniform magnetic field, the mirror ratio is unity and no particles are confined. As $R_{m} \rightarrow \infty$, the probability of escape goes to zero. Current technology restricts maximum magnetic field strengths in the mirror regions to well below $50 \mathrm{Tesla}^{(15)}$, hence the mirror ratio of the system will be limited to fairly low values. Loss probabilities are used in the simulation to modify the relativistic particle number densities at each time step. Plasma diffusion and end losses are negligible over millisecond plasma confinement times ${ }^{(10)}$, and the propellant number density is assumed to remain constant during confinement.

\section{Engine Performance}

Energy transfer efficiencies for the engine are calculated using

$$
\eta=\frac{\left(E_{i}+E_{e}\right) n_{p}}{(1877 M e V) n_{\bar{p}}}
$$

where $E_{i}$ and $E_{e}$ are the calculated plasma ion and electron energies, respectively, $n_{p}$ is the hydrogen propellant number density, $n_{p}$ is the initial antiproton number density, and $1877 \mathrm{MeV}$ is the amount of energy released in each proton-antiproton annihilation.

A simple energy balance may be used to estimate the rise in propellant temperature due to the influx of annihilation energy:

$$
\eta M_{a} c^{2}=M_{p} c_{r} \Delta T
$$

where $M_{a}$ is the total amount of annihilated mass, $M_{p}$ is the total amount of propellant mass, $c_{v}$ is the specific heat of the propellant at constant volume, $\Delta T$ is the change in propellant temperature, and $\eta$ is the efficiency with which the annihilation energy is transferred to the propellant. In the limiting 
cases of low temperature molecular hydrogen and high temperature atomic or ionized hydrogen, the specific heat may be calculated using ${ }^{(16)}$

$$
c_{v}=\frac{R_{0}}{M(\gamma-1)}
$$

where $R_{0}$ is the universal gas constant, $M$ is the molecular (atomic) weight of the propellant, and $\gamma$ is the adiabatic index of the gas. For molecular hydrogen, $\gamma=7 / 5$, while for atomic or ionized hydrogen, $\gamma=5 / 3$. Using the energy balance equation, the plasma ion energies predicted by the code are converted to propellant temperatures via

$$
T(e V)=E_{\text {code }}(e V)(\gamma-1) M^{\prime}
$$

where both $T$ and $E_{\text {code }}$ are expressed in units of $e V$, and $M^{\prime}$ is the molecular or atomic weight in amu.

Number densities are used in the simulation to provide flexibility in the choice of total reaction chamber volume. Once the chamber volume $V_{c}$ is selected, the hydrogen propellant mass flow rate may be calculated from

$$
\dot{M}_{p}=\frac{m_{p} n_{p} V_{c}}{\Delta t}
$$

where $m_{p}$ is the atomic mass of hydrogen, $n_{p}$ is the hydrogen propellant number density, and $\Delta t$ is the pulse repetition period of the engine. Similarly, the antiproton mass flow rate may be found using

$$
\dot{M}_{\bar{p}}=\frac{m_{\bar{p}} n_{\bar{p}} V_{c}}{\Delta t}
$$

where $m_{p}$ and $n_{p}$ are the antiproton rest mass and initial number density, respectively.

Engine thrust and specific impulse are estimated using the ideal rocket approximations ${ }^{(17)}$. An additional assumption is made that all of the ionized exhaust leaves the chamber axially with the same average energy, directed by a $100 \%$ efficient magnetic nozzle. The exhaust velocity is then given by

$$
v_{e}=1.4 \times 10^{6} \sqrt{E_{\text {code }}(\mathrm{eV})} \frac{\mathrm{cm}}{\mathrm{s}}=1.4 \times 10^{6} \sqrt{\frac{T(\mathrm{eV})}{M(\gamma-1)}} \frac{\mathrm{cm}}{\mathrm{s}}
$$

and the estimated specific impulse $\left(I_{s p}\right)$ of the engine is

$$
I_{s p}=\frac{v_{e}}{980 \mathrm{~cm} / \mathrm{s}^{2}} s
$$

The equivalent continuous thrust produced during each pulse is given by

$$
T_{h}=\dot{M}_{p} v^{\prime}
$$

and in terms of propellant number density may be written

$$
T_{h}=\frac{m_{p} n_{p} v_{c}}{\Delta t_{e}} v_{e}
$$

where $\Delta t_{e}$ is the engine pulse period. 


\section{Results and Discussion}

The model was tested against an antiproion powered orbital-transfer vehicle (OTV) study performed by Cassenti ${ }^{(18)}$. The predictions of engine thrust and specific impulse ${ }^{(10)}$ agree with Cassenti's Monte Carlo based simulations to within 5\%. In addition, preliminary Monte Carlo simulations performed at the Jet Propulsion Laboratory (JPL) of the annihilation energy transfer efficiencies in a hydrogen plasma are in good agreement with the energy transfer efficiencies predicted by the code ${ }^{(19)}$.

Selected results are presented in the following sections for low, moderate, and high hydrogen propellant number densities and a range of antiproton number densities. Cases presented in the first part of each section assume no charged particles are lost from the system, and represent an upper limit on engine performance. Combinations leading to optimum propellant heating in each case are reexamined using finite magnetic mirror ratios to evaluate the effect of particle losses on engine performance. Mirror ratios of 2 and 3 are used in compliance with current technology constraints ${ }^{(16)}$. Antiproton and hydrogen injection energies are set at $0.1 \mathrm{eV}$ to allow rapid injection into the reaction chamber, yet retain a sufficiently low center-of-mass velocity for efficient annihilation. Maximum electron and ion energies, annihilation energy transfer efficiencies, minimum magnetic field strength reqirements, predicted specific impulse, and predicted thrust (normalized to reaction chamber volume and pulse repetition rate) are tabulated in each section, and estimates of engine performance are given.

\section{Low Number Density Hydrogen Plasma}

The lowest hydrogen propellant number density considered in this study is $10^{14} \mathrm{~cm}^{-3}$. Figures $3(\mathrm{a})$ through 3(d) display antiproton, pion, muon, and relativistic electron/positron number density evolutions for initial antiproton number densities between $10^{9} \mathrm{~cm}^{-3}$ and $10^{12} \mathrm{~cm}^{-3}$, respectively, and mirror ratios of infinity. It is seen that antiproton annihilation becomes less efficient as the initial antiproton number density is increased, due to the rapid heating of the hydrogen propellant and the resulting decrease in proton-antiproton annihilation cross sections. The fraction of antiprotons remaining in the system after one millisecond increases from essentially zero at lower antiproton number densities to almost $70 \%$ for initial antiproton number densities of $10^{12} \mathrm{~cm}^{-3}$. Charged annihilation and decay byproduct evolutions are altered by the prolonged annihilation times. Significant amounts of pions and muons remain in the system for longer times, and maximum byproduct number densities are reduced by the prolonged annihilation period. Electron/positron number densities, which increase to roughly 3 times the initial antiproton number density in Figure 3(a), are reduced to approximately $98 \%$ of the initial antiproton number density in Figure 3(d).

Plasma ion energies are plotted for each of the intial antiproton number densities in Figure 4. Comparing the number density evolutions with the temperature curves, it is clear that the electrons and positrons are primarily responsible for heating the plasma, with little or no contribution from the pions and muons. The continued production of particles during the prolonged annihilation times slowed program execution considerably, and computer runs were terminated at $1 \mathrm{~ms}$ confinement times for economy. Test runs performed out to $5 \mathrm{~ms}$ indicate that plasma temperatures increase linearly with increasing confinement time.

Balancing plasma temperature with efficient antiproton annihilation, the optimal antiproton number density for running the low hydrogen propellant number density engine appears to be around $10^{10} \mathrm{~cm}^{-3}$. This case was rerun using magnetic mirror ratios of 2 and 3 to evaluate the effect of charged particle losses on the heating process. As seen in Figure 5, the ion energy at $1 \mathrm{~ms}$ is reduced from approximately $8 \mathrm{eV}$ with no mirror losses to $4.5 \mathrm{eV}$ with a mirror ratio of 3 , and $3 \mathrm{eV}$ with a 


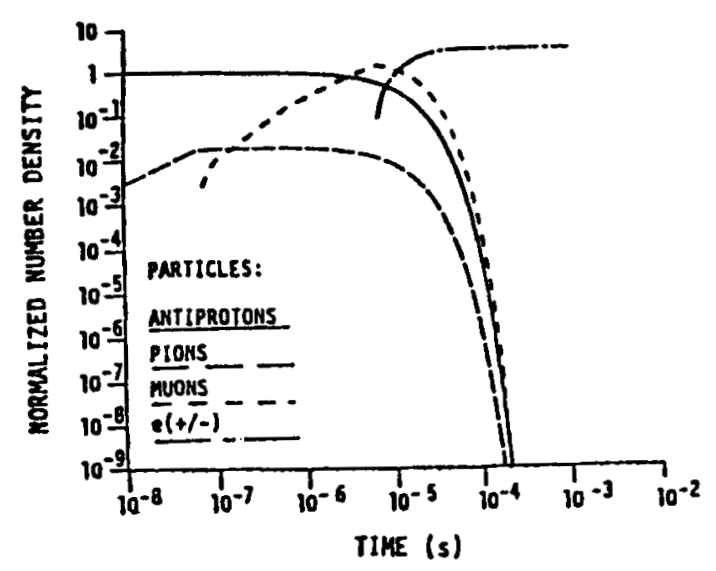

(a)

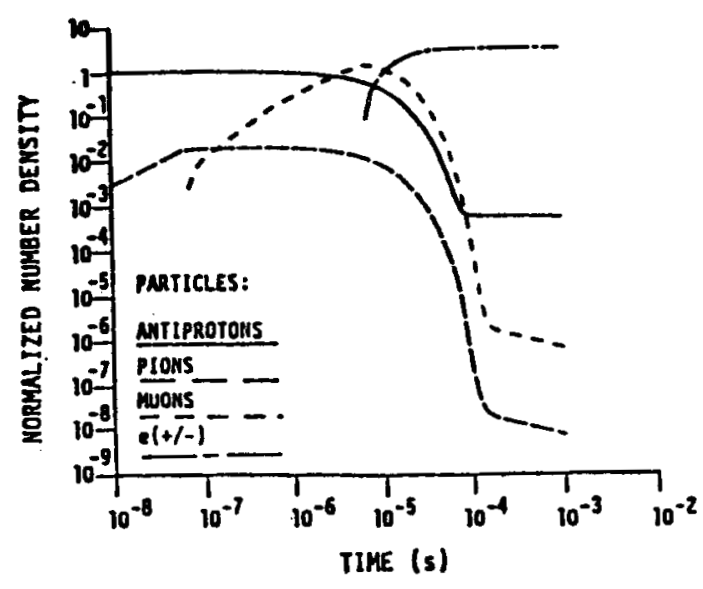

(b)

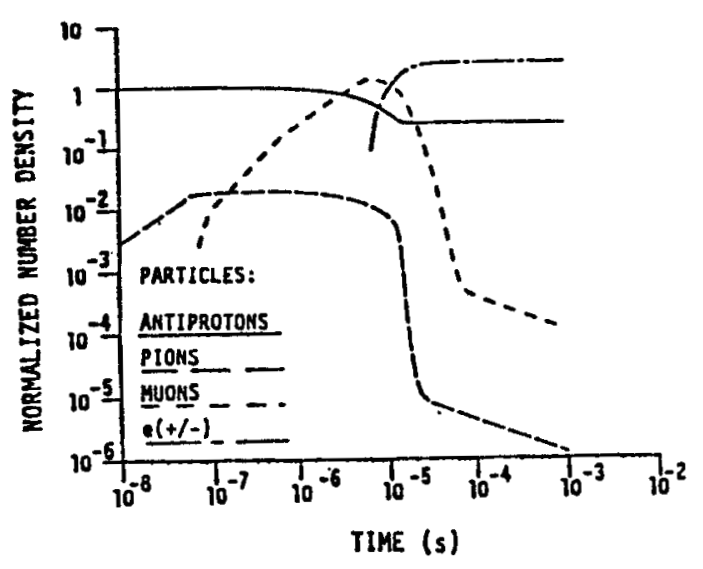

(c)

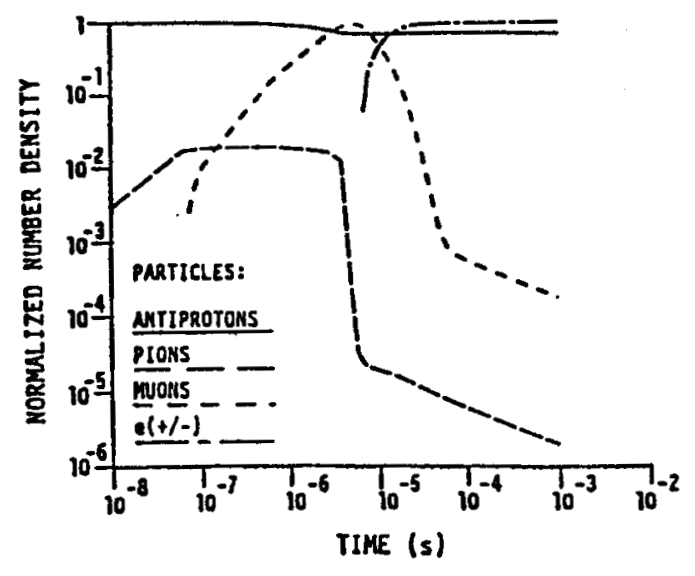

(d)

Figure 3: $\bar{P}, \pi, \mu$, and $e^{ \pm}$number density evolutions for $n_{p}=10^{14} \mathrm{~cm}^{-3}$. (a) $n_{p}=10^{\theta} \mathrm{cm}^{-3},(b) n_{\bar{p}}=$ $10^{10} \mathrm{~cm}^{-3}$, (c) $n_{p}=10^{12} \mathrm{~cm}^{-3}$, and (d) $n_{p}=10^{12} \mathrm{~cm}^{-3}$. No mirror losses. 


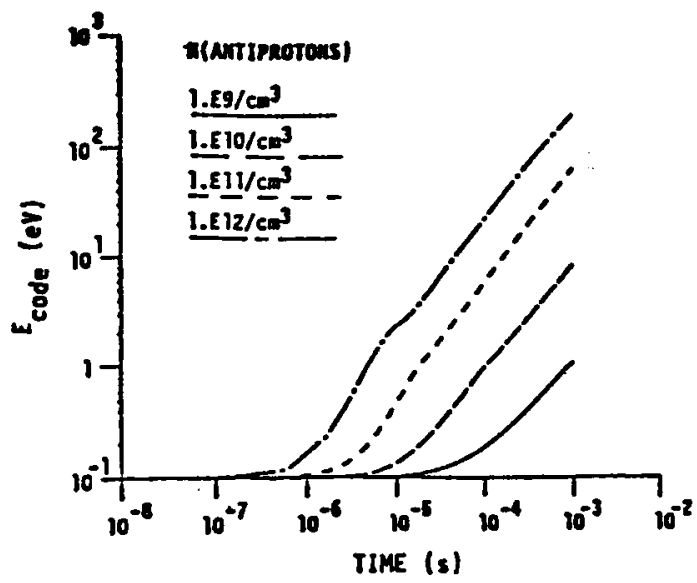

Figure 1: lon temperature versus time for various initial antiprolon number densities; $\mathrm{D}_{7}=10^{14} \mathrm{~cm}^{-3}$. No mirtor losses.

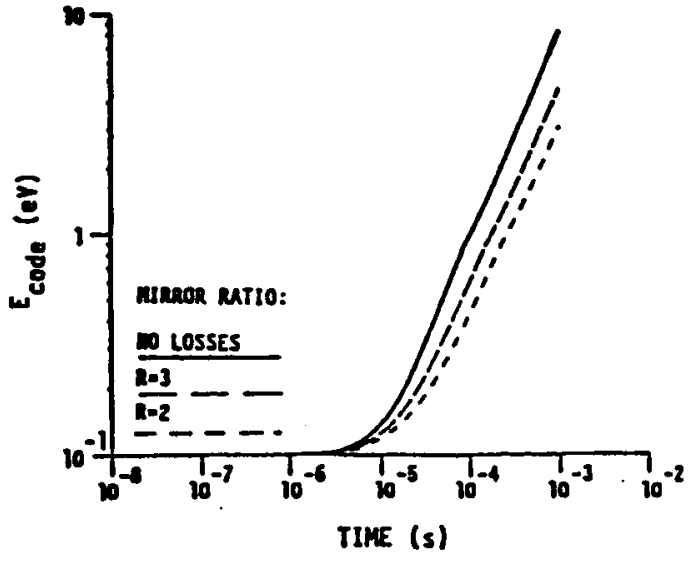

Figure 5: lon temperature versus time for various mirror ratios; $n_{p}=10^{14} \mathrm{~cm}^{-3}, n_{p}=10^{10} \mathrm{~cm}^{-2}$.

mirror ratio of 2 . The continual loss of pions from the system reduces muon production, which in turn reduces electron/positron production. The reduced electron/positron production rate, coupled with their continual loss from the magnetic mirror system, significantly diminishes the propellant temperature and the associated engine performance.

Table 2 summarizes the results for the low number density hydrogen plasma engine. Maximum propellant temperatures are achieved at higher antiproton number densities, but at the expense of wasting antiproton fuel. In addition, the plasma electrons are being heated faster than they can share their energy with the ions, and the plasma moves away from thermal equilibrium as the initial antiproton number density is increased. The optimum antiproton number density is estimated to be $10^{10} \mathrm{~cm}^{-3}$, and with a mirror ratio of 3 the maximum ion energy is about $4.5 \mathrm{eV}$ after $1 \mathrm{~ms}$, yielding a low annihilation energy transfer efficiency of $0.0048 \%$. Assuming a $5 \mathrm{~ms}$ confinement time, the ion temperature is $22.5 \mathrm{eV}$, and the efficiency is approximately $0.024 \%$. The calculated magnetic field strengths required for confinement are smaller than the 10 Tesla field required to constrain the pion gyroradii to reasonable values $(\approx 10 \mathrm{~cm})$, and are primarily due to relativistic particle kinetic pressure rather than plasma thermal pressure.

For the optimum case outlined above, a $1 \mathrm{~ms}$ plasma confinement time yields a specific impulse of 3030 seconds and a normalized thrust of $5.1 \times 10^{-10} \mathrm{~N} \cdot \mathrm{s} / \mathrm{cm}^{3}$, where the thrust has been normalized to the reaction chamber volume and engine pulse period. Assuming the reaction chamber has a radius of 1 meter and a length of 10 meters, and assuming a pulse period of $10 \mathrm{~ms}$, the equivalent continuous thrust is only $1.6 \mathrm{~N}(0.35 \mathrm{lbf})$. Increasing the plasma confinement time to $5 \mathrm{~ms}$ yields a specific impulse of 5530 seconds and an equivalent continuous thrust of $3.6 \mathrm{~N}(0.80 \mathrm{lbf})$. In both cases the hydrogen propellant flow rate is $3.1 \times 10^{21} \mathrm{H} / \mathrm{pulse}(525 \mathrm{mg} / \mathrm{s})$, and the antiproton flow rate is $3.1 \times 10^{17} \bar{p} /$ pulse $(52.5 \mu \mathrm{g} / \mathrm{s})$, since thrust is normalized to engine repetition rate and not plasma confinement time.

The low energy transfer efficiencies associated with the low hydrogen propellant number density severely limit the potential performance of the plasma engine. Increasing the hydrogen number density results in higher energy transfer efficiencies and improves engine performance, as shown in the following section. 


\begin{tabular}{|c|c|c|c|c|c|c|c|}
\hline$n_{p}\left(\mathrm{~cm}^{-3}\right)$ & $\mathrm{R}_{m}$ & $\mathrm{E}_{\max }^{e}(\mathrm{eV})$ & $\mathrm{E}_{\max }^{p}(\mathrm{eV})$ & $\eta$ & $\mathrm{B}_{\min }(\mathrm{T})$ & $\mathrm{I}_{\mathrm{op}}(\mathrm{s})$ & $\mathrm{Th}\left(\mathrm{N} \cdot \mathrm{sec} / \mathrm{cm}^{8}\right)$ \\
\hline $10^{9}$ & $\infty$ & 1.0 & 1.0 & $1.07 \times 10^{-4}$ & 0.35 & 1430 & $2.3 \times 10^{-10}$ \\
$10^{10}$ & $\infty$ & 8.1 & 8.1 & $8.5 \times 10^{-5}$ & 1.1 & 4040 & $6.7 \times 10^{-10}$ \\
$10^{10}$ & 3 & 4.5 & 4.5 & $4.8 \times 10^{-5}$ & 0.81 & 3030 & $5.1 \times 10^{-10}$ \\
$10^{10}$ & 2 & 3.0 & 3.0 & $3.2 \times 10^{-5}$ & 0.65 & 2475 & $4.1 \times 10^{-10}$ \\
$10^{11}$ & $\infty$ & 66 & 60 & $6.71 \times 10^{-5}$ & 3.1 & 11100 & $1.8 \times 10^{-8}$ \\
$10^{12}$ & $\infty$ & 370 & 190 & $3.0 \times 10^{-5}$ & 8.5 & 19700 & $3.3 \times 10^{-\theta}$ \\
\hline
\end{tabular}

Table 2: Plasma heating and rocket performance parameters for hydrogen number density of $10^{14} \mathrm{~cm}^{-3}$. Confinement time $=1 \mathrm{~ms}$; energy and efficiency parameters increase linearly with time for longer confinement times. Thrust is normalized to chamber volume and engine pulse period.

\section{Moderate Number Density Hydrogen Plasma}

A hydrogen propellant number density of $10^{16} \mathrm{~cm}^{-3}$ was chosen to model the performance of a moderate number density hydrogen plasma engine. Figures $6(a)$ and $6(b)$ show the antiproton and annihilation byproduct number density evolutions for initial antiproton number densities of $10^{9} \mathrm{~cm}^{-3}$ and $10^{12} \mathrm{~cm}^{-3}$, respectively. Number density evolutions for initial antiproton number densities of $10^{10} \mathrm{~cm}^{-3}$ and $10^{11} \mathrm{~cm}^{-3}$ are similar to those shown in Figure $6(\mathrm{a})$. At the lower antiproton number densities, essentially complete antiproton annihilation take place during the 5 ms confinement time. Pion number densities reach a maximum slightly in excess of the initial antiproton number densities before decaying from the system. Muon number densities climb to nearly 3 times the initial antiproton number densities before decaying, in agreement with the initial assumption of three charged mesons produced per annihilation event. The electron/positron number densities grow to 3 times the initial antiproton number density as the muons decay, and remain constant at this level. Increasing the initial antiproton number density to $10^{12} \mathrm{~cm}^{-3}$ heats the hydrogen too rapidly, decreasing the annihilation cross sections and prolonging the period of proton-antiproton annihilation. At the end of $5 \mathrm{~ms}$, nearly $10^{6} \mathrm{~cm}^{-3}$ antiprotons remain in the system, and will be exhausted with the plasma propellant.

Figure 7 shows the ion energy evolution for each of the initial antiproton number densities. The flattening of the energy curve for the case $n_{\tilde{p}}=10^{9} \mathrm{~cm}^{-3}$ is due to plasma bremsstrahlung; the energy deposited in the plasma by the charged annihilation products is balanced by bremsstrahlung losses, with no net gain in plasma energy. Energy deposition rates associated with the higher initial antiproton number densities exceed the rate at which bremsstrahlung radiation is lost, resulting in steadily increasing propellant temperatures. Also evident is the increased plasma energies caused by the relativistic muons and, to a lesser extent, the relativistic pions. At higher initial antiproton number densities the muons may substantially heat the plasma before decaying from the system.

Minimum magnetic field profiles for each of the above cases are presented in Figure 8. In each case, the minimum calculated field strength is due primarily to the relativistic particle kinetic pressure, which greatly exceeds the plasma thermal pressure. The evolution of the ninimum magnetic field strength closely follows the production and decay of the annihilation byproducts (Figure 6 ). 


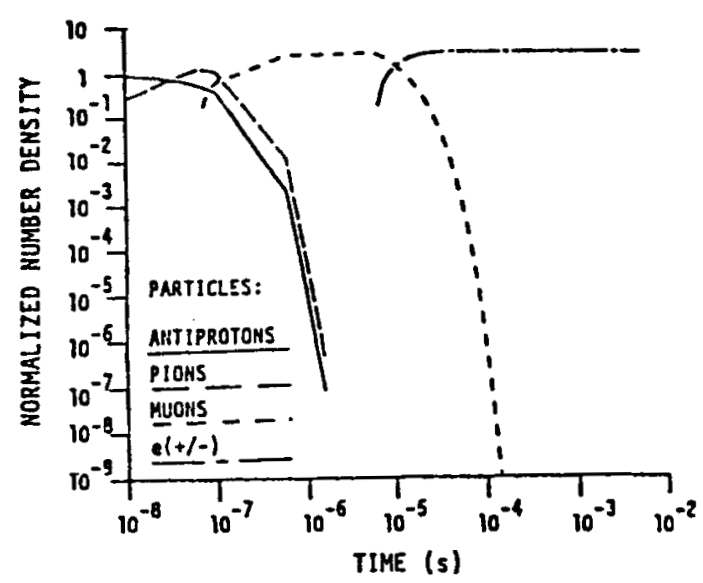

(a)

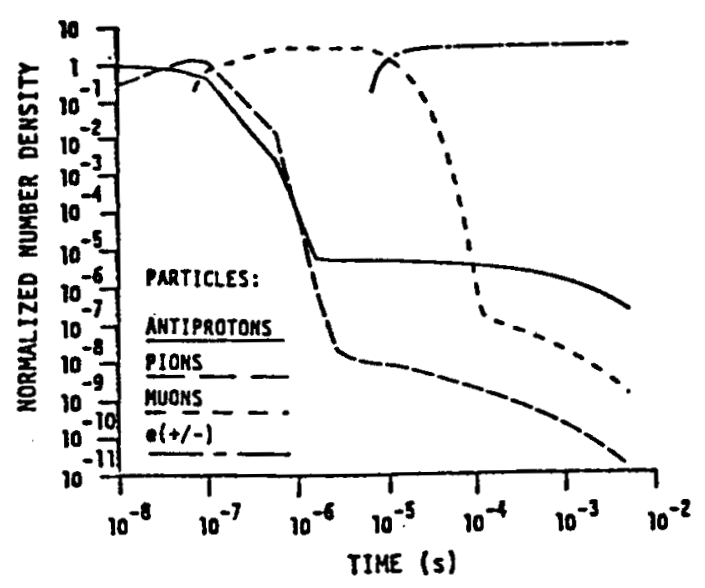

(b)

Figure 6: $P, \pi, \mu$, and $e^{ \pm}$number density erolutions for $n_{p}=10^{16} \mathrm{~cm}^{-3}$. (a) $\mathrm{n}_{p}=10^{\circ} \mathrm{cm}^{-3},(\mathrm{~b}) \mathrm{n}_{p}=$ $10^{12} \mathrm{~cm}^{-3}$. No nirtor losses.

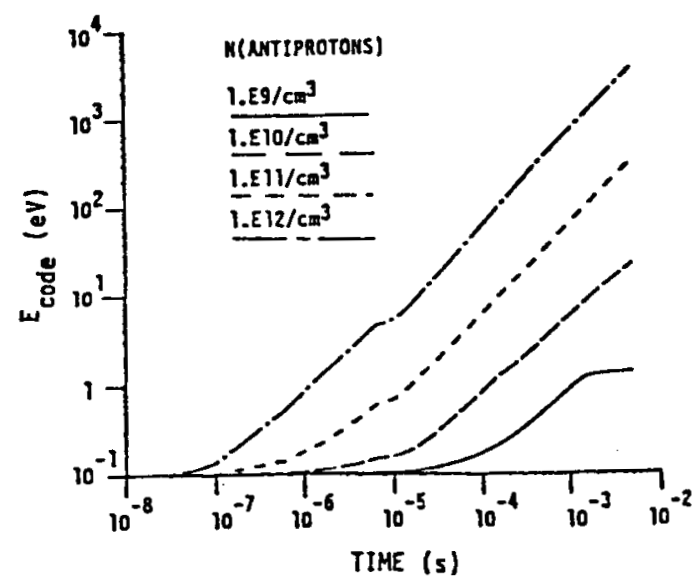

Figure $\mathrm{i}$ : lon temperature versus time for various initial antiproton number densities; $n_{p}=10^{16} \mathrm{~cm}^{-3}$. No mirtor losses.

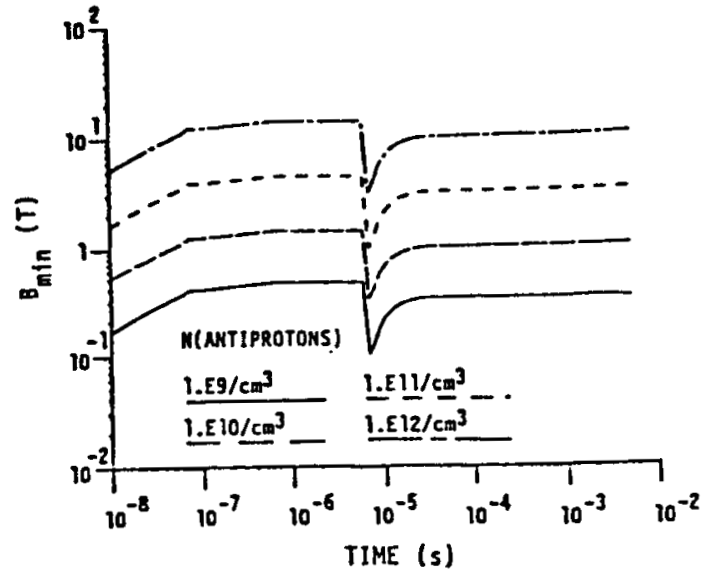

Figure 8: Minimum required magnetic field strength versus time for various initial antiproton number densities; $n_{p}=10^{16} \mathrm{~cm}^{-3}$, no mirror losses. 
For initial antiproton number densities of $10^{12} \mathrm{~cm}^{-3}$, the minimum field strength required for particle containment is approximately 14 Tesla, larger than the 10 Tesla previously assumed for reduced pion gyroradii. Rerunning this case with finite mirror ratios reduced the minimum required field strengths to 12 Tesla for a mirror ratio of 3 , and 10 Tesla for a mirror ratio of 2 (Figure 9 ). The decrease in ion energies due to the escape of charged particles from the confinement system is shown in Figure 10. Ion energies at $5 \mathrm{~ms}$ decrease from nearly $3280 \mathrm{eV}$ with no mirror losses to 1765 $\mathrm{eV}$ with $R_{m}=3$, and $1125 \mathrm{eV}$ with $R_{m}=2$.

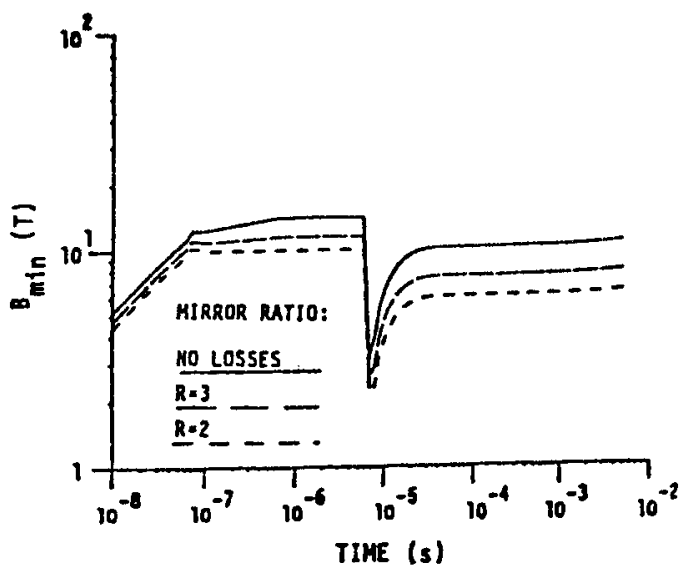

Figure 9: Minimum required magnetic field strength versus time for various mirror ratios; $n_{p}=10^{16} \mathrm{~cm}^{-3}$, $n_{p}=10^{12} \mathrm{~cm}^{-2}$.

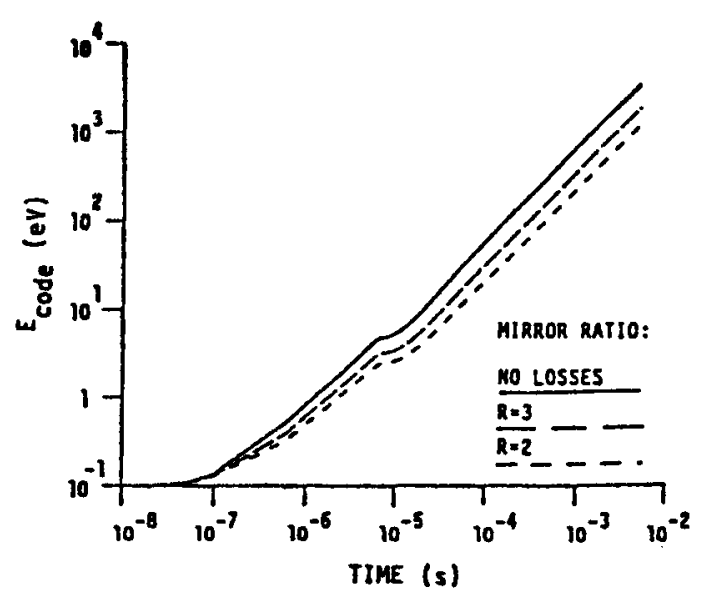

Figure 10: Ion temperature versus time for various mirror ratios; $\mathrm{n}_{p}=10^{16} \mathrm{~cm}^{-3}, \mathrm{n}_{p}=10^{12} \mathrm{~cm}^{-3}$

Table 3 lists plasma and engine performance parameters for the moderate number density plasma engine. Energy efficiencies are greatly improved over the low number density cases presented previously, and complete antiproton annihilation occurs under most operating conditions. A respectable range of thrust and specific impulse values are available for reasonable antiproton mass injection rates and magnetic mirror ratios, which may allow some flexibility in mission designs.

Assuming a reaction chamber radius of 1 meter, length of 10 meters, and engine repetition rate of $10 \mathrm{~ms}$ yields a hydrogen propellant mass flow rate of $3.14 \times 10^{23} \mathrm{H} / \mathrm{pulse}(52.5 \mathrm{~g} / \mathrm{s})$. For an antiproton number density of $10^{10} \mathrm{~cm}^{-3}$, the antiproton mass flow rate is $3.14 \times 10^{17} \bar{p} /$ pulse $(52.5$ $\mu \mathrm{g} / \mathrm{s}$ ). Using a mirror ratio of 3 yields a maximum ion energy of $10.4 \mathrm{eV}$ over a $5 \mathrm{~ms}$ plasma confinement time. The predicted specific impulse is $\mathbf{4 6 1 0}$ seconds, and the normalized thrust is $7.6 \times 10^{-7} \mathrm{~N} \cdot \mathrm{s} / \mathrm{cm}^{3}$, which for the above parameters yields an equivalent continuous thrust of 2,400 $\mathrm{N}(535 \mathrm{lbf})$ each cycle with an energy transfer efficiency of $1.1 \%$. Increasing the initial antiproton number density to $10^{12} \mathrm{~cm}^{-3}$ requires an antiproton mass flow rate of $3.14 \times 10^{19} \bar{p} / \mathrm{s}(5.25 \mathrm{mg} / \mathrm{s})$, and results in a maximum ion energy of $1765 \mathrm{eV}$ with an energy transfer efficiency of $1.9 \%$. The specific impulse is raised to an excessive 60,000 seconds, and the normalized thrust is $9.8 \times 10^{-6}$ $\mathrm{N} \cdot \mathrm{s} / \mathrm{cm}^{3}$ for an equivalent continuous thrust of $30,400 \mathrm{~N}(6800 \mathrm{lbf})$. The specific impulse might be lowered, and the thrust increased, by mixing cold hydrogen gas with the hot plasma propellant before exhausting. 


\begin{tabular}{|c|c|c|c|c|c|c|c|}
\hline$n_{p}\left(\mathrm{~cm}^{-3}\right)$ & $\mathrm{R}_{m}$ & $\mathrm{E}_{\max }^{e}(\mathrm{eV})$ & $\mathrm{E}_{\max }^{p}(\mathrm{eV})$ & $\eta$ & $\mathrm{B}_{\min }(\mathrm{T})$ & $\mathrm{I}_{s p}(\mathrm{~s})$ & $\mathrm{Th}\left(\mathrm{N} \cdot \mathrm{sec} / \mathrm{cm}^{8}\right)$ \\
\hline $10^{9}$ & $\infty$ & 1.3 & 1.3 & 0.009 & 0.32 & 1630 & $2.7 \times 10^{-7}$ \\
$10^{10}$ & $\infty$ & 22 & 22 & 0.023 & 1.4 & 6700 & $1.1 \times 10^{-6}$ \\
$10^{10}$ & 3 & 10.4 & 10.4 & 0.011 & 0.75 & 4610 & $7.6 \times 10^{-7}$ \\
$10^{11}$ & $\infty$ & 295 & 294 & 0.031 & 4.4 & 24500 & $4.0 \times 10^{-6}$ \\
$10^{11}$ & 3 & 152 & 152 & 0.016 & 2.4 & 17600 & $2.9 \times 10^{-6}$ \\
$10^{12}$ & $\infty$ & 3470 & 3280 & 0.036 & 14 & 81800 & $1.3 \times 10^{-6}$ \\
$10^{12}$ & 3 & 1806 & 1765 & 0.019 & 12 & 60000 & $9.8 \times 10^{-6}$ \\
$10^{12}$ & 2 & 1140 & 1125 & 0.012 & 10 & 48000 & $7.9 \times 10^{-6}$ \\
\hline
\end{tabular}

Table 3: Plasma heating and rocket performance parameters for hydrogen number density of $10^{16} \mathrm{~cm}^{-3}$. Confinement time $=5 \mathrm{~ms}$. Thrust is normalized to chamber volume and engine pulse period.

Although plasma bremsstrahlung radiation becomes a concern in terms of shielding requirements, the general performance of the antiproton powered plasma engine is dramatically improved using a moderate number density hydrogen propellant. The following section discusses predicted engine performance when the propellant number density is increased further.

\section{High Number Density Fydrogen Plasma}

Based on the previous results, it is expected that increasing the hydrogen number density will increase both the energy transferred into the plasma by the charged annihilation products and the energy lost from the plasma by radiation. The highest hydrogen number density considered in the study is $10^{18} \mathrm{~cm}^{-3}$, and Figures $11(\mathrm{a})$ and $11(\mathrm{~b})$ display charged particle number density evolutions corresponding to initial antiproton number densities of $10^{10} \mathrm{~cm}^{-3}$ and $10^{13} \mathrm{~cm}^{-3}$, respectively. The nearly identical figures bracket the range of antiproton number densities used in the high propellant number density simulations. Complete antiproton annihilation occurs within 100 nanoseconds in all cases, and each of the pion, muon, and electron/positron number densities in turn climb to maximum values 3 times higher than the initial antiproton number density. Due to the brief but intense antiproton annihilation period, the pions completely decay from the system within microseconds, and the muons are depleted within fractions of a millisecond, leaving the electrons and positrons to heat the plasma over the remaining confinement times.

The ion energy evolution (Figure 12) displays several remarkable features. At low initial antiproton number densities, the ion energy is limited by plasma bremsstrahlung radiation to values slightly over $1 \mathrm{eV}$. Increasing the initial antiproton number density to $10^{11} \mathrm{~cm}^{-3}$ raises the ion energy only slightly to $1.8 \mathrm{eV}$, again due to plasma radiation losses balancing the annihilation energy deposition. Increasing the initial antiproton number density still further results in a substantial heating of the plasma, as the energy deposited by the charged annihilation products exceeds the plasma energy lost by radiation. The role of the pions and mons in heating the plasma becomes nore evident, 
although it is interesting to note that the net energy deposition from these particles is limited by plasma bremsstrahlung to fairly low values. The bulk of the plasma heating comes from the electrons and positrons, which dominate the plasma radiation losses and raise the propellant temperature several orders of magnitude in a short period of time. Each of the energy curves are seen to peak at a time of $400 \mu \mathrm{s}$, after which the plasma energy falls rapidly to below $1 \mathrm{eV}$. A consideration of the energy loss equations shows that at this time the relativistic electrons and positrons have given up essentially all of their energy to the plasma, and with no source of heating the plasma rapidly loses energy by bremsstrahlung radiation. As the plasma cools it recombines (Figure 13), and confinement may be lost as the neutral hydrogen escapes through the magnetic fields. Consequently, to run the engine efficiently at high propellant number densities requires that the injection, heating, and exhaust cycle all be performed within $400 \mu \mathrm{s}$, or plasma cooling and neutral propellant loss will diminish the performance of the engine.

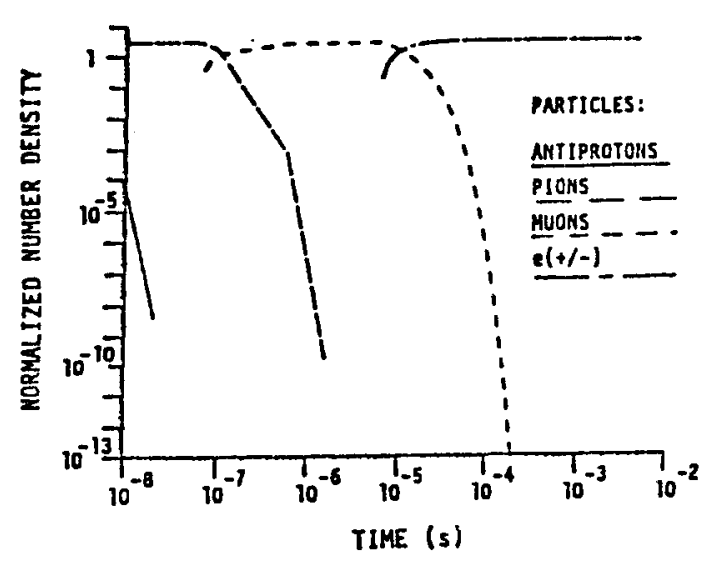

(a)

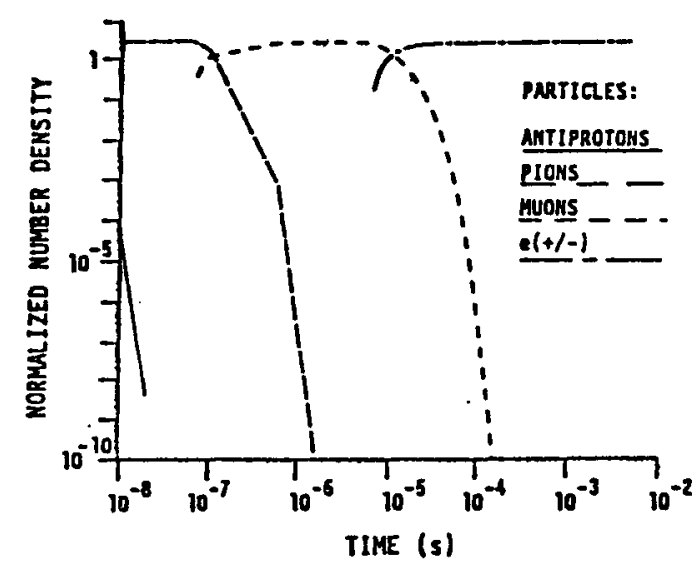

(b)

Figure 11: $\bar{P}_{,} \pi, \mu$, and $e^{ \pm}$number density evolutions for $\mathrm{n}_{p}=10^{18} \mathrm{~cm}^{-3}$. (a) $\mathrm{n}_{p}=10^{10} \mathrm{~cm}^{-3},(\mathrm{~b})$ $n_{p}=10^{13} \mathrm{~cm}^{-3}$. No mirror losses.

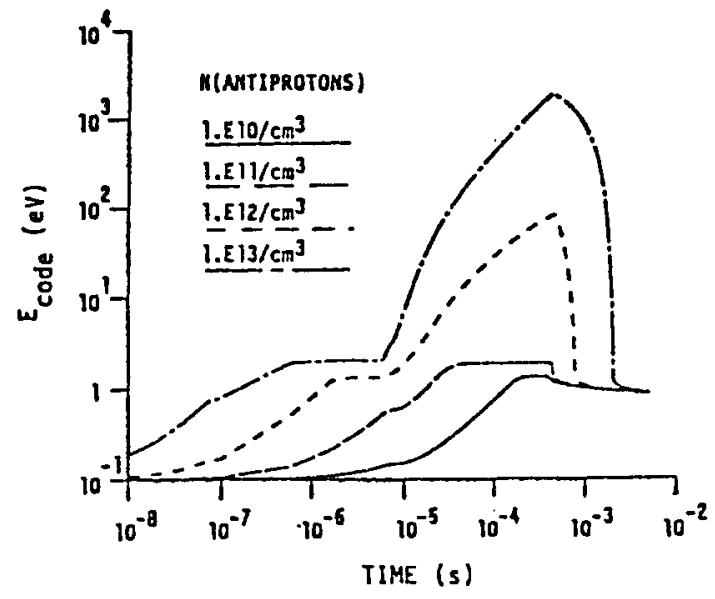

Figure 12: Ion temperature versus time for various initial antiprolon number densities; $n_{p}=10^{18} \mathrm{~cm}^{-3}$. No mirror losses.

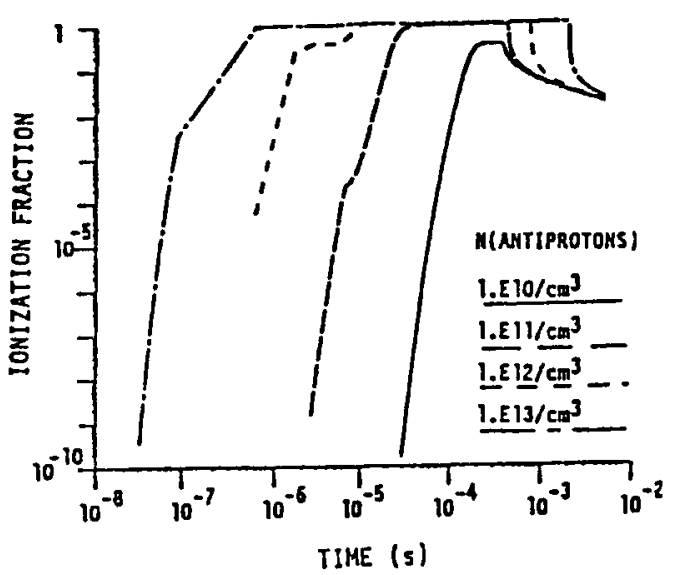

Figure 13: Fraction of $10^{18} \mathrm{~cm}^{-3}$ hydrogen gas ionized versus time for various initial antiproton number densities. No mirror losses. 
Figure 14 shows the minimum magnetic field strengths required to confine the system of charged particles and plasma, where again the field evolutions closely follow the production and decay of the relativistic annihilation byproducts. At low antiproton number densities, the field strengths are below the 10 Tesla field minimum set by the particle orbit constraints. As the initial antiproton number density is increased to $10^{12} \mathrm{~cm}^{-3}$, the minimum field increases to 18 Tesla. The evolution of the magnetic field strengths highlights the production and decay of the charged annihilation products, as well as the heating and subsequent cooling of the hydrogen plasma. Inereasing the initial antiproton number density to $10^{13} \mathrm{~cm}^{-3}$ requires a minimum confining field strength of nearly 60 Tesla for the central field coils, clearly beyond the capability of current or projected magnetic containment technology. Thus the optimum antiproton number density at which to run the high propellant number density plasma engine is around $10^{12} \mathrm{~cm}^{-3}$; higher antiproton number densities require unrealistic magnetic field strengths to confine the system, and plasma heating due to lower antiproton number densities is severely diminished by plasma bremsstrahlung radiation. Plasma and rocket performance parameters are summarized for the high number density propellant engine in Table 4 below.

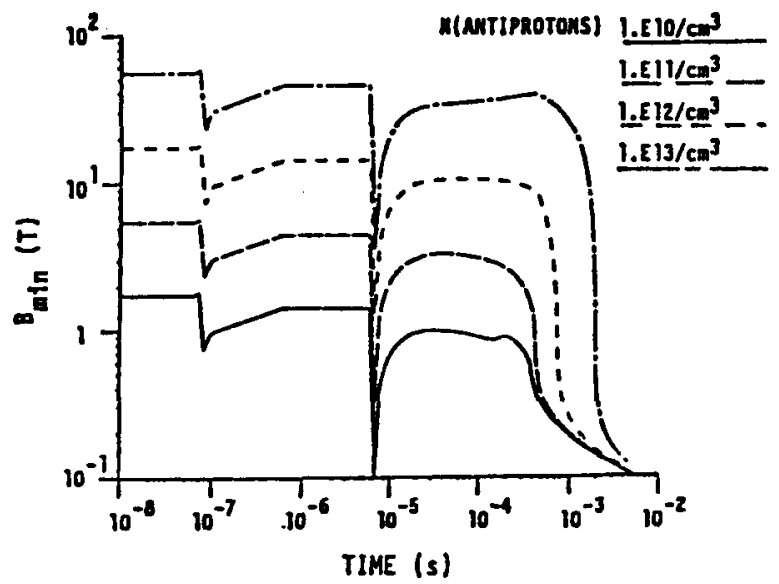

Figure 14: Minimum required magnetic field strength versus time for various initial antiproton number densities; $\mathrm{n}_{\mathrm{p}}=10^{18} \mathrm{~cm}^{-3}$, no mirror losses.

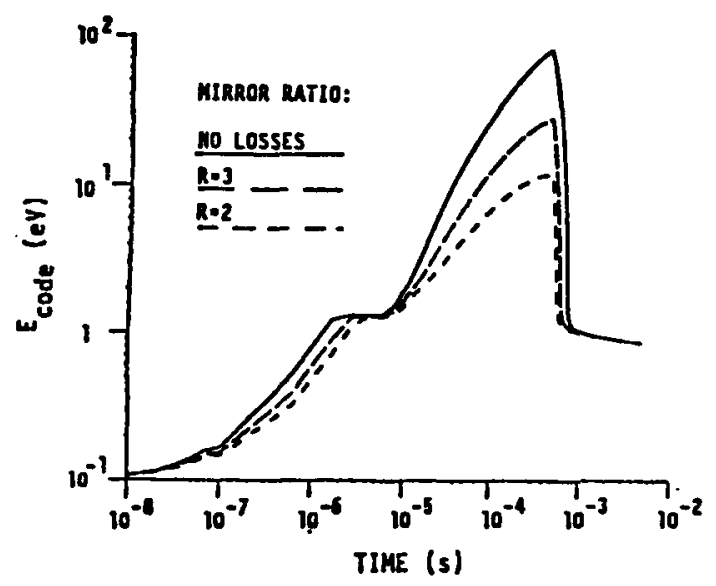

Figure 15: Ion temperature versus time for various mirrot ratios; $n_{p}=10^{18} \mathrm{~cm}^{-8}, n_{p}=10^{12} \mathrm{~cm}^{-3}$. 


\begin{tabular}{|c|c|c|c|c|c|c|c|}
\hline$n_{p}\left(\mathrm{~cm}^{-3}\right)$ & $\mathrm{R}_{m}$ & $\mathrm{E}_{\max }^{e}(\mathrm{eV})$ & $\mathrm{E}_{\max }^{p}(\mathrm{eV})$ & $\eta$ & $\mathrm{B}_{\min }(\mathrm{T})$ & $\mathrm{I}_{\mathrm{p}}(\mathrm{s})$ & $\mathrm{Th}\left(\mathrm{N} \cdot \mathrm{sec} / \mathrm{cm}^{3}\right)$ \\
\hline $10^{10}$ & $\infty$ & 1.3 & 1.3 & 0.139 & 1.8 & 1630 & $2.7 \times 10^{-5}$ \\
$10^{11}$ & $\infty$ & 1.8 & 1.8 & 0.019 & 5.7 & 1920 & $3.1 \times 10^{-5}$ \\
$10^{12}$ & $\infty$ & 81 & 81 & 0.086 & 18 & 12860 & $2.1 \times 10^{-4}$ \\
$10^{12}$ & 3 & 28 & 28 & 0.030 & 15.7 & 7560 & $1.2 \times 10^{-4}$ \\
$10^{12}$ & 2 & 12 & 12 & 0.012 & 14.6 & 4950 & $8.1 \times 10^{-5}$ \\
$10^{13}$ & $\infty$ & 1780 & 1780 & 0.189 & 57 & 60300 & $9.9 \times 10^{-4}$ \\
\hline
\end{tabular}

Table 4: Plasma heating and rocket performance parameters for hydrogen number density of $10^{18} \mathrm{~cm}^{-3}$. Confinement time $=400 \mu \mathrm{s}$. Thrust is normalized to chamber volume and engine pulse period.

The maximum ion energy reaches $81 \mathrm{eV}$ for the optimum case with no particle losses. The ion energy is reduced to $28 \mathrm{eV}$ for a mirror ratio of 3 , and to $12 \mathrm{eV}$ for a mirror ratio of 2 (Figure 15). The corresponding magnetic field strengths are reduced from 18 Tesla with no mirror losses to 16 Tesla when $R_{m}=3$ and 15 Tesla when $R_{m}=2$, indicating that the relativistic charged particle pressure rather than the plasma thermal pressure plays a dominant role in defining the minimum magnetic field strengths. Assuming a mirror ratio of 2 , the specific impulse is 4950 seconds, and the normalized engine thrust is $8.1 \times 10^{-5} \mathrm{~N} \cdot \mathrm{s} / \mathrm{cm}^{3}$. Assuming the standard $1 \mathrm{~m}$ by $10 \mathrm{~m}$ reaction chamber and a $10 \mathrm{~ms}$ engine repetition rate, and assuming the propellant is exhausted at its peak temperature within $400 \mu \mathrm{s}$, yields an equivalent continuous thrust of $250 \mathrm{kN}$ (56 klbf) with an energy transfer efficiency of $1.2 \%$.

\section{Additional Design Considerations}

There are an abundance of basic technology issues related to the design and operation of an antiproton powered vehicle, not the least of which concerns the amount of antiprotons required for fuel (current yearly production rates would suffice for only a few engine pulses). Adopting the uncertain premise that the antihydrogen fuel will be available, several crucial design elements remain to be evaluated.

\section{Magnetic Field Coils}

The magnetic mirror system used to contain the charged, relativistic annihilation products and the ionized plasma is presumed to consist of superconducting solenoidal field coils for the central chamber, bracketed at each end with superconducting magnetic mirror coils for plasma stability and confinement. One end of the magnetic mirror system is pulsed, allowing the heated propellant to escape. The remaining mirror coil and the main solenoidal field coils would be run steady state, and could conceivably be energized using space station facilities before a mission begins. An efficient energy storage and transfer system will be required to power the exhaust coil during pulsed operation and return the energy to storage when the field is relaxed. One possibility is to store the energy inductively using additional superconducting electromagnets. Stacey ${ }^{(20)}$ discusses the use of 
a capacitive shunt transfer circuit to transfer energy between a superconducting storage coil and a superconducting fusion device coil. The energy transfer circuit and related pulse forming network could be adapted for a pulsed mirror coil with minimal modifications.

The energy transfer between the pulsed mirror coil and the energy storage coil will undoubtedly be less than $100 \%$ efficient, and an onboard energy source may be required to replenish lost field coil energy. One possible source of auxiliary power is the charged particle flux leaking through the magnetic mirrors during each heating cycle. It may be possible to use a direct energy converter placed at the end of the mirror system to tap some of the directed charged energy as the pions (and other charged particles) escape magnetic confinement. Even a very inefficient converter might provide enough energy to resupply the pulsed mirror coil, and the energy of the annihilation products escaping through the loss cones would not be entirely wasted. Magnetic field coil power requirements and auxiliary power alternatives are discussed in reference 10.

The mass of the magnetic confinement system is expected to be a major part of the total rocket mass. Superconducting coils made of $\mathrm{Nb}_{3} \mathrm{Sn} / \mathrm{NbTi}$ are currently used in magnetic fusion engineering ${ }^{(21)}$, and are used here to provide a preliminary estimate of the total coil mass which may be required for an antiproton powered vehicle. The density of NbTi is approximately $6.4 \mathrm{~g} / \mathrm{cm}^{3}$, and coil dimensions may be scaled from the MARS (Mirror Advanced Reactor Study) tandem nirror fusion plant design ${ }^{(21)}$. The central cell of the MARS design consists of 44 identical solenoidalal coils spaced 3.16 meters apart. Each coil has an inner radius of $2.44 \mathrm{~m}$, thickness of $0.932 \mathrm{~m}$, and width of $0.884 \mathrm{~m}$, and provides a central field strength of roughly $5 \mathrm{~T}$. The antiproton powered rocket annihilation chamber has half the radius but requires twice the field, hence the coil thickness and width may remain about the same. The coil volume is approximately $7.6 \mathrm{~m}^{3}$, corresponding to a mass of about $4.9 \times 10^{4} \mathrm{~kg}$. The assumed length of the antiproton annihilation chamber is 10 meters; spaced 3 meters apart, the main chamber would require 3 solenoidal coils. Combined with the two end coils, the estimated mass of the magnet system is roughly $2.5 \times 10^{5} \mathrm{~kg}$. In practice, the coil structure includes copper stabilizing material, electrical insulation, mechanical support, and associated cooling systems, which will substantially increase the mass and may significantly diminish low thrust engine performance.

\section{Antiproton Storage and Transport}

Several anthors have discussed methods of storage and transport for the antihydrogen fuel ${ }^{(5,6)}$. As an added complication, storage and transport systems for the magnetically confined plasma engine must also contend with charged annihilation products and plasma particles escaping through the mirror coil back along the antiproton beam path. It may be beneficial to place the storage chamber at an angle with respect to the magnetic mirror axis to keep charged particles from penetrating and heating the storage region. Pulsed electric or magnetic guide fields could then be used to direct incoming antiproton bunches into the annihilation chamber at the start of each cycle. After injection the guide fields are pulsed off as charged particles from the resulting annihilations escape back along the axis, perhaps passing through the direct energy converter mentioned above to supply auxiliary power. When the reaction chamber is evacuated, the guide fields are pulsed on and a new bunch of antiprotons are diverted into the chamber. Alternatively, antiprotons might be recombined with positrons, and the neutral antihydrogen atoms injected across the field lines. Efficient antiproton fuel transport and injection cycling is critical for the pulsed plasma engine operation. 


\section{Shielding}

Copious anounts of radiation are produced both by antiproton annihilations and by the confined plasma. Sensitive ship components must be proteeted, and the edditional mass of a radiation shield will reduce the amount of payload the rocket can transport. Cassenti(22) has performed a preliminary numerical analyais of the crew shielding required for an antiproton powered OTV spacecraft ${ }^{(18)}$. The crew is assumed to be located 100 meters away from the radiation source, and a tungsten shadow shield is placed between the crew and the annihilation chamber. The radiation source is assumed to be the $200 \mathrm{MeV}$ (average energy) gamma rays produced by prompt neutral pion decay. The simulation models pair creation, ionisation energy loss, and positron annihilation production of secondary gamma rays within the shield material, but neglects the production of high energy electrons ereated by ionisation and Compton scattering of secondary particles. The high energy electrons will be a source of secondary gamma radiation due to bremsstrahlung emission, and the simulation underestimates the number of lower energy gamma rays produced in the shielding material. The simulation is one dimensional, hence radiation scattering is also neglected. Assuming $8 \mathrm{mg}$ of mass are annihilated, the model predicts a minimum shield thickness 0 $6.5 \mathrm{~cm}$ for adequate crew shielding. The estimated mass of the shield is thus on the order of $3.9 \times 10^{3} \mathrm{~kg}$. The 10 tonne payload assumed in the OTV study must now include this mass, reducing the deliverable payload by nearly $40 \%$.

Additional mass will be required to protect the supercoaducting coils, which are immediately outside the reaetion chamber. Based on the above radiation model, and assuming the coils are placed 1 meter from the radiation source, an additional $11.8 \mathrm{~cm}$ of tungsten shielding is required for the OTV magnetic field coils. Assuming a blanket shield is used around the entire reaction chamber, the required shield mass is $28 \times 10^{3} \mathrm{~kg}$, or nearly 3 times greater than the 10 tonne payload assumed in the OTV mission study. Shadow shielding of the superconducting coils could reduce the required shielding mass, although protecting the coils from scattered radiation may limit any potential mass reduction.

The problem is exacerbated for the antiproton powered plasma engine operating at high temperatures, since plasma bremsstrahlung radiation must now be included in the total dosage calculations. Unlike the penetrating $200 \mathrm{MeV}$ gammas, the lower energy bremsstrahlung photons will deposit most of their energy in the surface layers of the shield material, leading to wall ablation and shield degradation similar to that seen in magnetic fusion energy devices ${ }^{(20)}$. Presumably, much of the research directed toward solving the problem of first wall ablation in fusion plasma reactors can be transferred to the problem of shield ablation in the antiproton powered engine. The disparate energies of the gamma radiation may require a multilayered shield design, and the optimum design of such a shield is clearly an important area for future research.

A rough estimate of the maximum radiated energy from an antiproton powered plasma engine can be made by assuning an antiproton aumber density of $10^{12} \mathrm{~cm}^{-3}$, a hydragen plasma number density of $10^{18} \mathrm{~cm}^{-3}$, and a plasma temperature of $10 \mathrm{eV}$ (corresponding to the high propellant number density ease with a mirror ratio of 2). Again nssuming a chamber radius of $1 \mathrm{~m}$ and a length of $10 \mathrm{~m}$ yields a total chamber volume of $31 \mathrm{~m}^{3}\left(3.1 \times 10^{7} \mathrm{~cm}^{3}\right)$. The total gamma radiation produced in each $400 \mu$ s pulse by neutral pion decay is approximately

$$
E_{\gamma} \approx 10^{12} \cdot 3.1 \times 10^{\tau} \cdot 2 \cdot 2 \cdot 200 \mathrm{MeV} \approx 4.0 \mathrm{GJ}
$$

where it is assumed that 2 neutral pions are created in each annihilation event and each subsequently decays into two $200 \mathrm{MeV}$ gamma rays. The plasma radiates an additional $6.6 \times 10^{8} \mathrm{~J}$ each pulse, for a total radiated energy of $4.7 \mathrm{GJ}$ per pulse. Dividing by the chamber surface area yields a total dosage of about $75 \mathrm{MJ} / \mathrm{m}^{2}$ per pulse; an engine repetition rate of $10 \mathrm{msec}$ would thus produce 7.5 
$\mathbf{G W} / \mathrm{m}^{2}$. Assuming the superconducting coil cooling system can remove the equivalent of $\mathrm{a}$ few Watts $/ \mathrm{m}^{2}$ requires a shield thickness on the order of $17 \mathrm{~cm}$, for a total shield mass of $2.24 \times 10^{5} \mathrm{~kg}$. The equivalent continuous thrust produced by the engine is around $250 \mathrm{kN}$ over a $10 \mathrm{~ms}$ engine cycle. Assuming the shield mass dominates the total mass of the engine yields an average acceleration of about $1 \mathrm{~m} / \mathrm{s}^{2}$. To reach a velocity of $5.5 \mathrm{~km} / \mathrm{s}$ (suitable for an OTV mission) requires a burn time of approximately 5500 seconds (1.5 hours). The expended propellant mass is on the order of $2.9 \times 10^{4}$ $\mathbf{k g}$, and the required antiproton mass is 28.5 grams.

The shielding mass would be reduced for shorter reaction chamber lengths or for longer engine cycles, although such measures would also reduce the average engine thrust. Alternatively, the shield mass might be reduced by using shadow shields to protect the crew, superconducting coils, and related support structure. In either instance the shield mass will comprise a major portion of the total engine mass, a reality imposed on any antiproton powered engine using magnetic confinement. The impact of such severe mass penalties on mission performance underscores the need for refined radiation shield analysis.

Since the radiation shielding absorbs significant power, it must be cooled either actively or by passive radiation cooling. The additional mass of the cooling system will further reduce the available payload fraction, and design studies should be carried out to determine the optimum means of dissipating the energy imparted to the shield. It may be possible to mitigate the mass penalty associated with shielding and shield cooling by tapping some of the energy deposited in the shield for use in an auxiliary power system ${ }^{(22)}$. The increase in shield temperature caused by absorbing an amount of energy $E$ is given by

$$
\Delta T=\frac{E}{J_{c_{p}} M}
$$

where $c_{p}$ is the specific heat of the shield material $\left(33 \mathrm{cal} / \mathrm{kg}^{0} \mathrm{C}\right.$ for tungsten), $J$ is the mechanical equivalent of heat $(4.186 \mathrm{~J} / \mathrm{cal})$, and $M$ is the shield mass. The temperature of a $2.3 \times 10^{6} \mathrm{~kg}$ tungsten shield absorbing roughly $5 \mathrm{GJ} /$ pulse would increase about $160^{\circ} \mathrm{C}$ per pulse. Depending on the rate at which energy was removed from the shield, the steady state temperature could be much higher, providing a useful reservoir of energy for additional power requirements.

\section{Conclusion}

Matter-antimatter annihilation produces more energy per unit mass than any other method of energy production, making it an attractive energy source for spacecraft propulsion. The energetic charged particles produced in proton-antiproton annihilation may be exhausted directly to provide a low thrust, high specific impulse engine, or used to heat a propellant for higher thrust and lower specific impulse. The magnetically confined pulsed plasma engine is designed to minimize thermal constraints on material walls while providing a range of operating parameters. In this concept, antiproton beams are injec',ed axially into a pulsed magnetic unirror system, where they annihilate with an initially neutral hydrogen gas. The resulting charged annihilation products transfer energy to the hydrogen propellant, which is then exhausted through one end of the pulsed mirror system to provide thrust. Engine thrust and specific impulse are controlled by adjusting the number of hydrogen atoms and antiprotons injected each pulse.

Numerical simulations were developed to calculate the annihilation rate of antiprotons in hydrogen and to follow the resulting pion, muon, and electron/positron number density evolutions. Plasma heating due to charged particle interactions and cooling due to radiative losses were evaluated for several initial antiproton and hydrogen number densities. Mininum magnetic field strengths required to 
confine the plasma and charged annihilation products were evaluated as the system evolved. Plasma temperatures were used to estimate engine thrust and specific impulse.

The pulsed hydrogen plasma engine suffers from extremely low energy transfer efficiencies at low hydrogen number densities, and from excessive plasma bremsstrahlung radiation at high hydrogen number densities. Optimum performance was obtained with a hydrogen number density of $10^{16} \mathrm{~cm}^{-3}$ and antiproton number densities between $10^{10} \mathrm{~cm}^{-3}$ and $10^{12} \mathrm{~cm}^{-3}$. Low to moderate thrusts $(\leq 30$ $\mathrm{kN}$ ) over a wide range of specific impulse values ( 4600 seconds to 60,000 seconds) were generated using this moderate hydrogen number density for $5 \mathrm{~ms}$ plasma confinement times and realistic magnetic mirror systems. Energy transfer efficiencies were generally less than $2 \%$, with relativistic electrons and positrons providing most of the plasma heating. Higher thrust could be produced with higher hydrogen number densities, but the plasma will radiatively cool and recombine if it is not expelled within a few hundred microseconds after injection. Assuming the plasma can be fully exhausted from a realistic magnetic mirror system at its peak temperature, the high number density hydrogen propellant can produce a maximum specific impulse of $\mathbf{4 9 5 0}$ seconds with an equivalent continuous thrust of approximately $250 \mathrm{kN}$.

Apart from the formidable problem of generating and storing sufficient antiproton fuel, the key engineering issues facing the magnetically confined pulsed plasma engine are the design and construction of the superconducting magnetic field coils, efficient pulsed coil operation, antiproton transport into the annihilation region, and radiation shielding. Preliminary analysis indicates that substantial mass will be required to shield the superconducting coils from the high energy $\gamma$-rays emitted in the $p \bar{p}$ annihilation process. Shielding problems are compounded by plasma bremsstrahlung radiation, which will contribute to shield surface ablation and degradation. The mass of the radiation shield will dominate the total engine mass and significantly impact engine performance. These initial results underscore the need to include the radiation shield mass in all antiproton powered spacecraft designs.

\section{Acknowledgements}

This report is based on dissertation research performed at the University of New Mexico, Albuquerque, New Mexico. The author is indebted to Dr. Norman Roderick, Department of Chemical and Nuclear Engineering, UNM, for his counseling and guidance during the course of this project. Computer simulations were performed at the NASA Lewis Research Center, Cleveland, Ohio.

\section{References}

1. Massier, P. F., "The Need for Expanded Exploration of Matter-Antimatter Annihilation for Propulsion Application", Journal of the British Interplanetary Society (J.B.I.S.), Vol. 35, 1982, pp. 387-390.

2. Forward, R. L., B. N. Cassenti, and D. Miller, "C'ost C'omparison of C'hemical and Antihydrogen Propulsion Systems for High $\Delta V$ Missions", AIAA-85-1455, July 1985.

3. Sanger, E., "Zur theorie der Photoneraketen" ("The Theory of Photon Rockets"), IngeniturArchiv. Vol. 21, 1953, pp. 213-226.

4. Maj. G. Nordley, USAF, Air Force Astronautics Laboratory, Edwards AFB, CA; personal communication. 
5. Forward, R. L., Antiproton Annihilation Propulsion, AFRPL TR-85-034, AFRPL/LKC, Edwards AFB, C'a., Sep. 1985.

6. Morgan, D. L., "Concepts for the Design of an Antimatter Annihilation Rocket", J.B.I.S., Vol. 35,1982 , pp. 405-412.

7. Morgan, D. L. and V. M. Hughes, "Atomic Processes Involved in in Matter-Antimatter Annihilation", Phys. Rev., Vol. D-2 (8), 1970, pp. 1389-1399.

8. Agnew, L. E. Jr., T. Elioff, W. G. Fowler, R. L. Lander, W. M. Powell, E. Segre, H. M. Steiner, H. S. White, C. Wiegand, and T. Ypsilentis, "Antiproton Interactions in Hydrogen and Carbon Below $200 \mathrm{MeV}$ ", Phys. Rev. Vol. 118 (5), 1960, pp. 1371-1391.

0. Morgan, D. L., Annihilations of Antiprotons in Heavy Nuclei, AFRPL TR-86-011, AFRPL/LKc, Edwards AFB, Ca., April 1986.

10. LaPointe, M. R., "Antiproton Annihilation Propulsion with Magnetically Confined Plasma Engines", Ph.D. Dissertation, The University of New Mexico, Albuquerque, New Mexico, May 1989.

11. Sternheimer, R. M., "Fundamental Principles and Methods of Particle Detection", Methods of Experimental Physics: Nuclear Physics, Vol 5(A), L. C. L. Yuan and C.-S. Wu (eds.), Academic Press, 1961, pp. 1-288.

12. Sivukhin, D. V., "Coulomb Collisions in a Fully Ionized Plasma", Reviews of Plasma Physics, Vol. 4, M. A. Leontovich (ed.), Consultants Bureau, New York, 1966, pp. 93-240.

13. Post, R. F., "Experimental Base of Mirror-Confinement Physics", Fusion, Vol. 1(A): Magnetic Confinement, E. Teller (ed.), 1981, pp. 369-402.

14. Glasstone, S., and R. H. Lovberg, Controlled Thermonuclear Reactions, D. van Nostrand Campany, Inc., Princeton, N.J., 1960, pp. 366-400.

15. Montgomery, D. B. "High Field Magnetic Confinement of Fusion Plasmas", and Miura, N. and F. Herlach, "Pulsed and Ultrastrong Magnetic Fields", Topics in Applied Physics, Vol. 57: Strong and Vltrastrong Magnetic Fields and Their Applications, F. Herlach (ed.), Springer-Verlag, 1985, pp. 205-349.

16. Jahn, R. G., Physics of Electric Propulsion, McGraw Hill, Inc., 1968.

17. Sutton, G. P., Rocket Propulsion Elements, 2nd ed., J. Wiley \& Sons, 1958, pp. 45-53.

18. Cassenti, B. N., "Antimatter Propulsion for OTV Applications", J. Propulsion and Power, Vol. 1 (2), March-A pril, 1985, pp. 143-149.

19. Callas, J., "Modeling Antiproton - Plasma Interactions", presentation at Antiproton Technology Workshop, Brookhaven Nutional Laboratories, Upton, N.Y., May 1989.

20. Stacey, W. M., Fusion, J. Wiley \& Sons, 1984

21. Gross, R. A., Fusion Energy, J. Wiley \& Sons, Pub., 1984.

22. Cassenti, B. N., "Radiation Shield Analysis for Antimatter Rockets", United Technologies Research Center, Silver Lane, East Hartford, Connecticut 06108; 1987 United Technologies Corporation. 


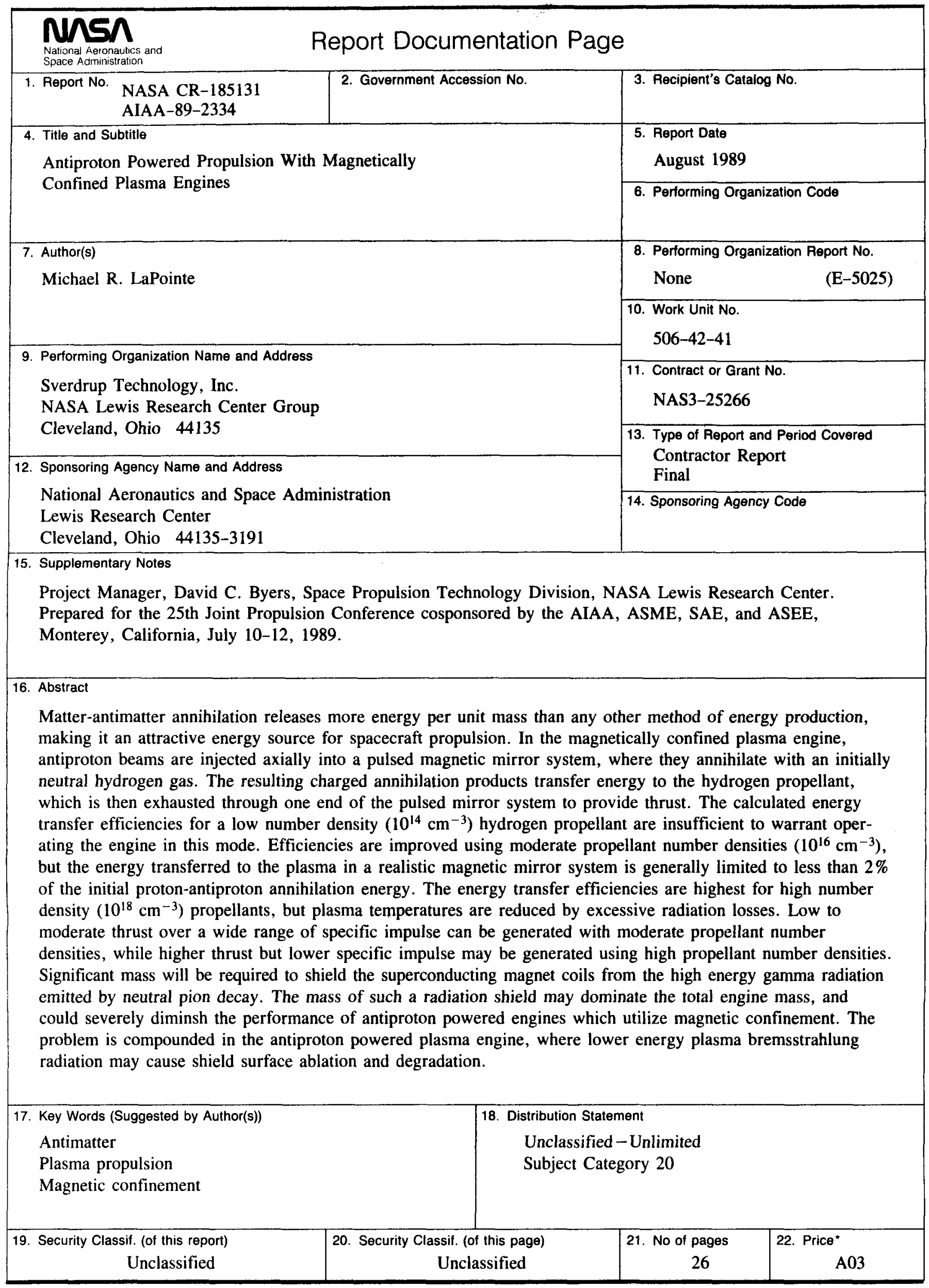

